\title{
A collagen-binding protein enables molecular imaging of kidney fibrosis in vivo
}

Citation for published version (APA):

Baues, M., Klinkhammer, B. M., Ehling, J., Gremse, F., van Zandvoort, M. A. M. J., Reutelingsperger, C. P. M., Daniel, C., Amann, K., Babickova, J., Kiessling, F., Floege, J., Lammers, T., \& Boor, P. (2020). A collagen-binding protein enables molecular imaging of kidney fibrosis in vivo. Kidney International, $97(3)$, 609-614. https://doi.org/10.1016/j.kint.2019.08.029

Document status and date:

Published: 01/03/2020

DOI:

10.1016/j.kint.2019.08.029

Document Version:

Publisher's PDF, also known as Version of record

Document license:

Taverne

Please check the document version of this publication:

- A submitted manuscript is the version of the article upon submission and before peer-review. There can be important differences between the submitted version and the official published version of record.

People interested in the research are advised to contact the author for the final version of the publication, or visit the DOI to the publisher's website.

- The final author version and the galley proof are versions of the publication after peer review.

- The final published version features the final layout of the paper including the volume, issue and page numbers.

Link to publication

\footnotetext{
General rights rights.

- You may freely distribute the URL identifying the publication in the public portal. please follow below link for the End User Agreement:

www.umlib.nl/taverne-license

Take down policy

If you believe that this document breaches copyright please contact us at:

repository@maastrichtuniversity.nl

providing details and we will investigate your claim.
}

Copyright and moral rights for the publications made accessible in the public portal are retained by the authors and/or other copyright owners and it is a condition of accessing publications that users recognise and abide by the legal requirements associated with these

- Users may download and print one copy of any publication from the public portal for the purpose of private study or research.

- You may not further distribute the material or use it for any profit-making activity or commercial gain

If the publication is distributed under the terms of Article $25 \mathrm{fa}$ of the Dutch Copyright Act, indicated by the "Taverne" license above, 


\title{
A collagen-binding protein enables molecular imaging of kidney fibrosis in vivo
}

\author{
Maike Baues ${ }^{1,12}$, Barbara M. Klinkhammer ${ }^{2,3,12}$, Josef Ehling ${ }^{1,2}$, Felix Gremse ${ }^{1}$, \\ Marc A.M.J. van Zandvoort ${ }^{4}$, Chris P.M. Reutelingsperger ${ }^{5}$, Christoph Daniel $^{6}$, Kerstin Amann ${ }^{6}$, \\ Janka Bábíčková ${ }^{3,7,8}$, Fabian Kiessling ${ }^{1}$, Jürgen Floege ${ }^{3}$, Twan Lammers ${ }^{1,9,10}$ and Peter Boor 2,3,11
}

${ }^{1}$ Institute for Experimental Molecular Imaging, RWTH Aachen University Hospital, Aachen, Germany; ${ }^{2}$ Institute of Pathology, RWTH Aachen University Hospital, Aachen, Germany; ${ }^{3}$ Department of Nephrology and Immunology, RWTH Aachen University Hospital, Aachen, Germany; ${ }^{4}$ Department of Biochemistry, University Hospital Maastricht (azM), Maastricht, The Netherlands; ${ }^{5}$ Department of Pathology, Electron Microscopy Unit, University of Maastricht, Maastricht, The Netherlands; ${ }^{6}$ Institute of Pathology, University Erlangen, Erlangen, Germany; ${ }^{7}$ Institute of Molecular Biomedicine, Comenius University, Bratislava, Slovakia; ${ }^{8}$ Department of Clinical Medicine, University of Bergen, Bergen, Norway; ${ }^{9}$ Department of Pharmaceutics, Utrecht University, Utrecht, The Netherlands; ${ }^{10}$ Department of Targeted Therapeutics, University of Twente, Enschede, The Netherlands; and ${ }^{11}$ Electron Microscopy Facility, RWTH Aachen University Hospital, Aachen, Germany

Pathological deposition of collagen is a hallmark of kidney fibrosis. To illustrate this process we employed multimodal optical imaging to visualize and quantify collagen deposition in murine models of kidney fibrosis (ischemiareperfusion or unilateral ureteral obstruction) using the collagen-binding adhesion protein CNA35. For in vivo imaging, we used hybrid computed tomographyfluorescence molecular tomography and CNA35 labeled with the near-infrared fluorophore Cy7. Upon intravenous injection, CNA35-Cy7 accumulation was significantly higher in fibrotic compared to non-fibrotic kidneys. This difference was not detected for a non-specific scrambled version of CNA35-Cy7. Ex vivo, on kidney sections of mice and patients with renal fibrosis, CNA35-FITC co-localized with fibrotic collagen type I and III, but not with the basement membrane collagen type IV. Following intravenous injection, CNA35-FITC bound to both interstitial and perivascular fibrotic areas. In line with this perivascular accumulation, we observed significant perivascular fibrosis in the mouse models and in biopsy sections from patients with chronic kidney disease using computer-based morphometry quantification. Thus, molecular imaging of collagen using CNA35 enabled specific non-invasive quantification of kidney fibrosis. Collagen imaging revealed significant perivascular fibrosis as a consistent component next to the more commonly assessed interstitial fibrosis. Our results lay the basis for further probe and protocol optimization towards the clinical translation of molecular imaging of kidney fibrosis.

\footnotetext{
Correspondence: Peter Boor, Institute of Pathology, RWTH Aachen University Hospital, Pauwelsstrasse 30, 52074 Aachen, Germany. E-mail: pboor@ukaachen.de; or Twan Lammers, Institute for Experimental Molecular Imaging, RWTH Aachen University Hospital, Forckenbeckstrasse 55, 52074 Aachen, Germany. E-mail: tlammers@ukaachen.de

${ }^{12}$ Equal contribution.
}

Received 21 May 2019; revised 2 August 2019; accepted 22 August 2019; published online 18 September 2019
Kidney International (2020) 97, 609-614; https://doi.org/10.1016/ j.kint.2019.08.029

KEYWORDS: chronic kidney disease (CKD); collagen; extracellular matrix; molecular imaging; non-invasive imaging; renal fibrosis

Copyright (C) 2019, International Society of Nephrology. Published by Elsevier Inc. All rights reserved.

\section{Translational Statement}

The pathological correlate of chronic kidney disease is renal fibrosis, that is, deposition of the extracellular matrix, in particular collagen. Here we introduced molecular imaging for noninvasive visualization and quantification of collagen deposition in murine renal fibrosis models using the collagen binding molecular probe CNA35 in combination with hybrid computed tomography-fluorescence molecular tomography imaging. For translation into clinical use, further probe and protocol optimization will be required. The final goal is to establish noninvasive diagnostic methods to specifically monitor and quantify kidney fibrosis, to provide novel end points for clinical trials, and to improve the management of patients with chronic kidney disease.

R enal fibrosis is a common finding in chronic kidney disease, independent of the underlying disease etiology. ${ }^{1}$ Fibrosis is characterized by the pathological production and accumulation of the extracellular matrix in the renal interstitium, mainly comprising collagen. Although multiple antifibrotic targets have been identified in preclinical research, no targeted therapeutics have made it into clinical routine. A potential reason for this poor translation is the lack of noninvasively accessible fibrosis-specific study end points in clinical trials. ${ }^{2,3}$ We recently showed that elastin-specific magnetic resonance imaging enables noninvasive staging and treatment monitoring of renal fibrosis. ${ }^{4}$ Here, we describe a method for the visualization and quantification of the most common extracellular matrix component in fibrotic kidneys, 
that is, collagen. We used the collagen-binding protein fragment collagen-binding adhesion protein 35 (CNA35), which binds to fibril-forming collagen but not to other extracellular matrix components (such as laminin, elastin, and fibronectin) and which has already been shown to hold promise for visualizing cardiovascular fibrosis. ${ }^{5-7}$ Using optical imaging, we demonstrate in 2 murine models-unilateral ureteral obstruction (UUO) and ischemia-reperfusion injury-that fluorophore-labeled CNA35 can specifically and noninvasively detect collagen deposition in renal fibrosis.

\section{RESULTS}

\section{Optical imaging of CNA35 accumulation in fibrotic kidneys}

Hybrid computed tomography-fluorescence molecular tomography imaging showed significantly higher CNA35 accumulation in kidneys with ischemia-reperfusion injuryinduced fibrosis than in contralateral healthy kidneys at 24, 48, and 72 hours after i.v. injection of $1 \mathrm{nmol}$ of CNA35-Cy7 (Figure 1a and b). Off-target accumulation of CNA35 was observed in the liver and spleen, which likely results from capture and clearance by the reticuloendothelial system (Supplementary Figure S1A). Clearance of CNA35 from fibrotic kidneys was substantially slower than that from healthy kidneys. Similar findings were observed after i.v. injection of $5 \mathrm{nmol}$ of CNA35-Cy7 (Supplementary Figure S1B and C). Ex vivo fluorescence reflectance imaging confirmed the in vivo findings, showing significantly increased probe retention in fibrotic versus healthy kidneys for both 1 and 5 nmol of CNA35-Cy7 (Figure 1c and d and Supplementary Figure S1D and E). To demonstrate that CNA35 specifically targets collagen in fibrotic kidneys, we injected a Cy7-labeled scrambled version of CNA35. As opposed to CNA35-Cy7, the scrambled probe did not specifically accumulate in fibrotic kidneys and it was cleared equally fast from fibrotic and healthy kidneys (Figure 1e). To verify the experimental setup, we confirmed that the expression levels of collagen type I and III in fibrotic kidneys were comparable in all mice included in the analysis (Supplementary Figure S2).

\section{Microscopic confirmation of the collagen-binding specificity of CNA35}

To prove the specificity of CNA35 binding to collagen fibers, we performed costainings with fluorescein isothiocyanate (FITC)-labeled CNA35 and different types of collagen on renal tissue sections from healthy mice and mice with UUO. CNA35-FITC showed high colocalization with the scar collagen type I and III in fibrotic mouse kidneys, but overlapped minimally with the basement membrane collagen type IV (Figure 2a). We validated these findings in kidney biopsy specimens from patients with chronic kidney disease (Supplementary Figure S3). As in murine kidneys, CNA35FITC was localized in the interstitium and around vessels, mainly colocalizing with collagen type I and III and hardly with type IV. Ex vivo analyses of renal tissues from mice with UUO injected with CNA35-FITC at 0.5, 4, and 24 hours after i.v. administration confirmed the specificity of the probe, showing significantly higher accumulation in fibrotic than in healthy kidneys (Figure $2 \mathrm{~b}$ and $\mathrm{c}$ ), and a staining pattern that clearly reflects perivascular binding to fibrillar collagen (Figure 2d).

\section{Collagen upregulation during renal fibrosis is enhanced in perivascular areas}

Given strong CNA35-FITC accumulation observed in perivascular areas (Figure 2d), we next quantified vascular alterations and perivascular fibrosis in mice with UUO and ischemia-reperfusion injury. Although the lumen of arcuate and interlobular arteries as well as afferent and efferent arterioles was markedly narrowed, the adventitia was significantly thickened by $>50 \%$ because of collagen deposition (Figure 3a-e). Collagen type III staining of human biopsies from patients with IgA nephropathy confirmed this pronounced perivascular fibrosis, which progressively increased with increasing chronic kidney disease stage (Figure 3f).

\section{DISCUSSION}

We demonstrate specific and noninvasive assessment of collagen deposition in renal fibrosis using fluorophore-labeled CNA35 and optical imaging. Our data extend previous studies with CNA35 in cardiovascular fibrosis, providing the first proof of concept for molecular imaging of collagen content in fibrotic kidneys. Ex vivo and histological analyses revealed that CNA35 mainly binds to the fibrillar scar collagen type I and III, which are the main components of the extracellular matrix in fibrotic kidneys, whereas only a low degree of colocalization was observed with nonfibrillar collagen type IV in basement membranes, consistent with solid-phase binding assays. ${ }^{5}$ Importantly, we found similar colocalization of CNA35 and collagen in murine and human tissues.

Our ex vivo optical imaging, assessing CNA35-FITC distribution after i.v. injection, highlighted a prominent perivascular fibrosis in murine models, supported by our quantitative histological analyses showing significant fibrotic thickening of the arterial adventitia due to collagen deposition in fibrosis, both in mice and in patients. This common and until now rather neglected feature of renal fibrosis is unlikely mediated by hypertension, because both ischemia-reperfusion injury and UUO models are normotensive. Our findings extend the current concept of perivascular cells as important mediators of renal fibrosis, particularly of $\mathrm{Glil}^{+}$cells, which reside in and spread from the arterial adventitia. ${ }^{8,9}$

CNA35 has 2 key advantages compared with other probes, such as radiolabeled antibodies, that can also be used to specifically target and image collagen deposition in fibrotic tissues. First, CNA35 is promiscuous in detecting several fibrillar collagen; and second, it has an $\sim 5$-fold smaller size, allowing better tissue penetration, in particular in fibrotic lesions. Other small peptide- and indole-based probes have recently been reported for specific noninvasive imaging of pulmonary fibrosis using positron emission tomography. ${ }^{10,11}$ These compounds may also hold significant potential for renal fibrosis staging and treatment monitoring, albeit none have yet been tested in kidneys. 

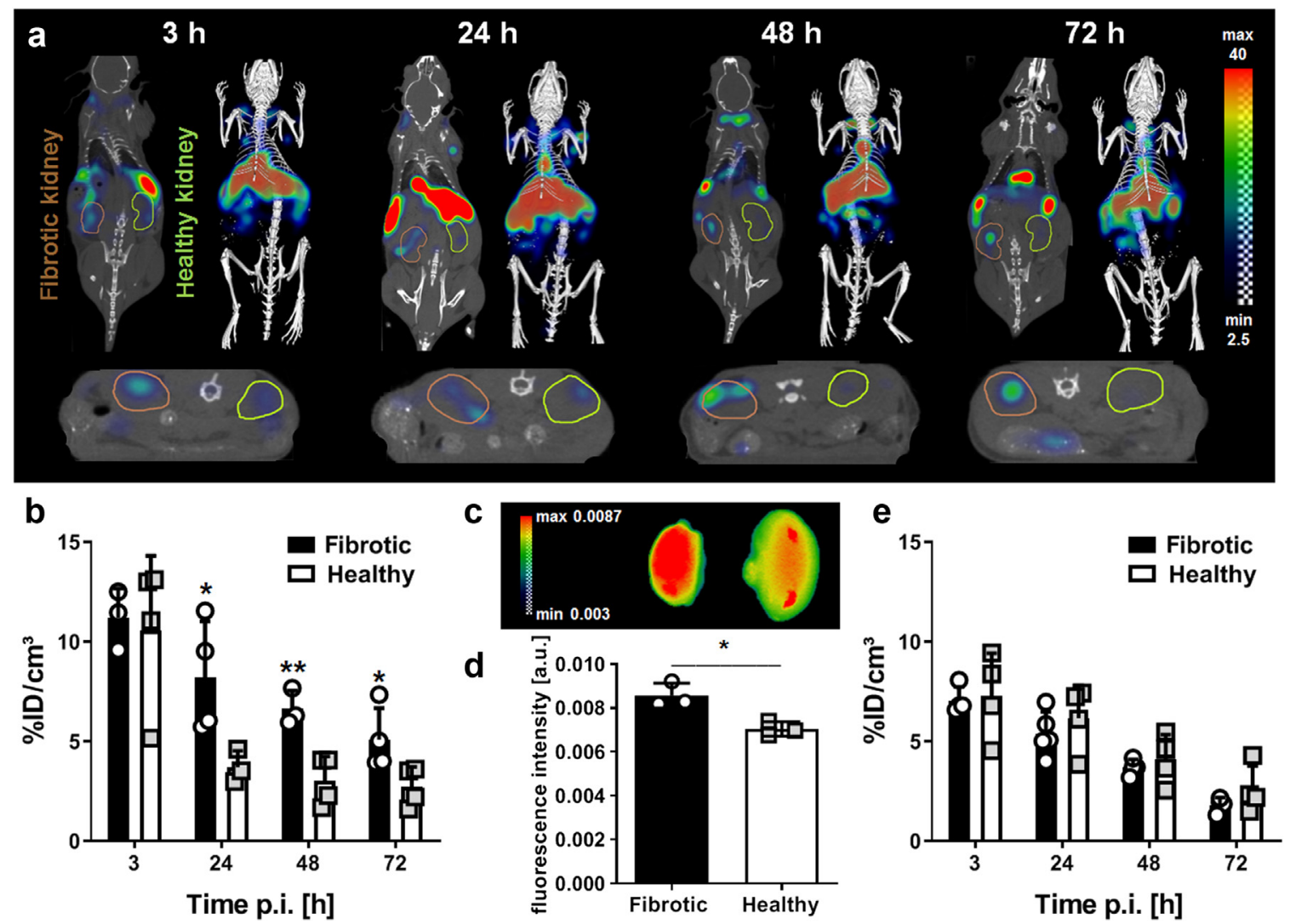

Figure 1 | In vivo and ex vivo optical imaging of collagen deposition in kidneys with ischemia-reperfusion injury (I/R)-induced fibrosis using CNA35-Cy7. (a) Coronal (left) and transversal (bottom) slices as well as 3-dimensional renderings (right) for hybrid computed tomography-fluorescence molecular tomography (CT-FMT) imaging of CNA35 biodistribution in a mouse with I/R-induced renal fibrosis at different time points after i.v. injection of $1 \mathrm{nmol}$ of CNA35-Cy7. The fibrotic kidney is encircled in brown (left) and the healthy kidney in green (right) in the coronal view. (b) Quantification of the in vivo CT-FMT images shows prominent accumulation of CNA35-Cy7 in fibrotic kidneys at 24, 48, and 72 hours after probe administration. (c,d) Ex vivo fluorescence reflectance imaging at 72 hours post-injection (p.i.) confirms higher CNA35 accumulation in fibrotic kidneys (left). (e) Quantification of the accumulation in kidneys of scrambled CNA35-Cy7, demonstrating no specific accumulation in fibrotic versus healthy kidneys. Data are mean \pm SD of 4 mice per group. ${ }^{*} P<0.05,{ }^{* *} P<0.01 . \% \mathrm{IC} / \mathrm{cm}^{3}$, percentage of the injected dose per cubic centimeter. To optimize viewing of this image, please see the online version of this article at www.kidney-international.org.

We recently reported noninvasive elastin-specific molecular magnetic resonance imaging of kidney fibrosis. ${ }^{4}$ Compared with elastin, collagen is much more abundant in fibrotic tissue, making it in principle easier to target. Collagen is also upregulated earlier in the disease course. Conversely, the higher baseline abundance of collagen in healthy kidneys and other target tissues may result in a measurable fluorescent signal in nondiseased kidneys and healthy tissue. Another major limitation of our study was the low animal numbers, which were based on sample size calculations from our previous studies and were, however, sufficient to show significant results. Furthermore, it was impossible to perform and analyze the imaging data in a truly blinded manner because of the obvious morphological distinction between healthy and fibrotic kidneys as well as the difference in CNA35 signal intensity. In the present study, we have not included experiments measuring different degrees of fibrosis, as we have previously demonstrated to be feasible using elastin-specific imaging. ${ }^{3}$ In addition, for a successful translation into the clinic, further contrast agent adaptation is needed before first-in-human studies, because optical imaging approaches are not suitable in kidney fibrosis.

Our study lays the foundation for future systematic experiments defining and optimizing targets, probes, and imaging algorithms for translational noninvasive and quantitative diagnosis, staging, and treatment monitoring of kidney fibrosis.

\section{METHODS}

Information on animal models, human renal samples, histology, immunohistochemistry, and immunofluorescence microscopy are provided in the Supplementary Methods.

\section{Imaging probes and protocols}

CNA35 was produced as described previously ${ }^{5}$ and labeled with Cy7 for in vivo and with FITC for ex vivo optical imaging. A scrambled version of CNA35, that is, with amino acid substitution Y175K, rendering it unable to bind to collagen, was used as a control. Hybrid computed tomography-fluorescence molecular tomography and fluorescence reflectance imaging were performed using an in-house optimized procedure. ${ }^{12,13}$ Hybrid computed 

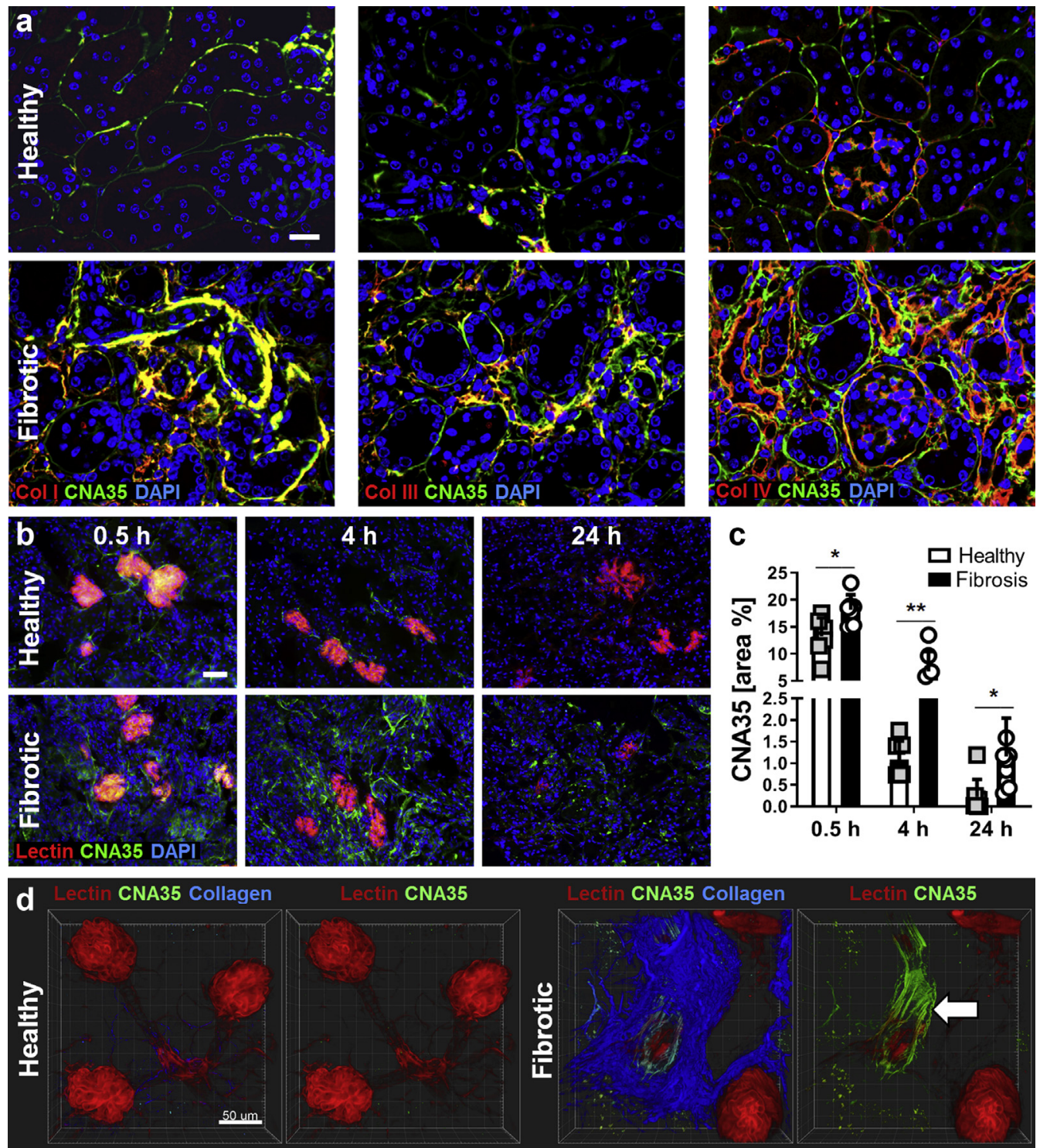

CNA35 Collagen

CNA35

Figure 2| Microscopy imaging of collagen deposition in fibrotic kidneys using CNA35-fluorescein isothiocyanate (FITC). (a)

Fluorescence microscopy analysis showing the colocalization of FITC-labeled CNA35 (green; upon incubation of tissue sections) with collagen fibers of type I, III, and IV (Col I, Col II, and Col III, respectively; all in red) on renal tissue sections from healthy mice and mice with unilateral ureteral obstruction. Nuclei are counterstained using 4',6-diamidino-2-phenylindole (DAPI; blue). Bar $=50 \mu \mathrm{m}$. (b) Representative immunofluorescent images showing the accumulation of FITC-labeled CNA35 (green) in healthy and fibrotic kidneys at 0.5, 4, and 24 hours after i.v. injection. Perfused blood vessels are counterstained using rhodamine-labeled lectin (red) and cell nuclei using DAPI (blue). Bar $=50 \mu \mathrm{m}$. (c) Quantification of CNA35-FITC accumulation in fibrotic versus healthy kidneys after i.v. injection. (d) Two-photon laser scanning microscopy indicated specific colocalization with collagen fibers (visualized using second harmonic generation) and much stronger enrichment of CNA35-FITC in fibrotic kidneys than in contralateral healthy kidneys, particularly around blood vessels (arrow). Bar $=50$ $\mu \mathrm{m}$. Data are mean $\pm \mathrm{SD}$ of kidney sections from 6 mice. ${ }^{*} P<0.05,{ }^{* *} P<0.01$. To optimize viewing of this image, please see the online version of this article at www.kidney-international.org.

tomography-fluorescence molecular tomography imaging was performed at multiple time points after i.v. injection of CNA35. The hybrid computed tomography-fluorescence molecular tomography data were analyzed using Imalytics Preclinical software (Gremse-IT $\mathrm{GmbH}$, Aachen, Germany). ${ }^{14}$ Organ segmentation based on the anatomical computed tomography data was used to quantify the fluorescence signal allocated to kidneys and other organs and expressed as the percentage of the injected dose per cubic centimeter. Organ accumulation was normalized to the total fluorescence intensity measured at 3 hours post-injection. At the end of the in vivo experiment, functional blood vessels were stained via i.v. injection of rhodamine-labeled lectin (Vector Laboratories, Burlingame, CA) to facilitate fluorescence and 2-photon microscopy.

\section{Statistical analysis}

All data are presented as mean $\pm \mathrm{SD}$. Paired $t$ tests were used to compare fibrotic and contralateral kidneys in 1 animal. An unpaired $t$ test was used for comparison between 2 groups. One-way analysis of variance followed by Bonferroni correction was used for comparing $>2$ groups. Statistical significance was defined as $P<0.05$. 

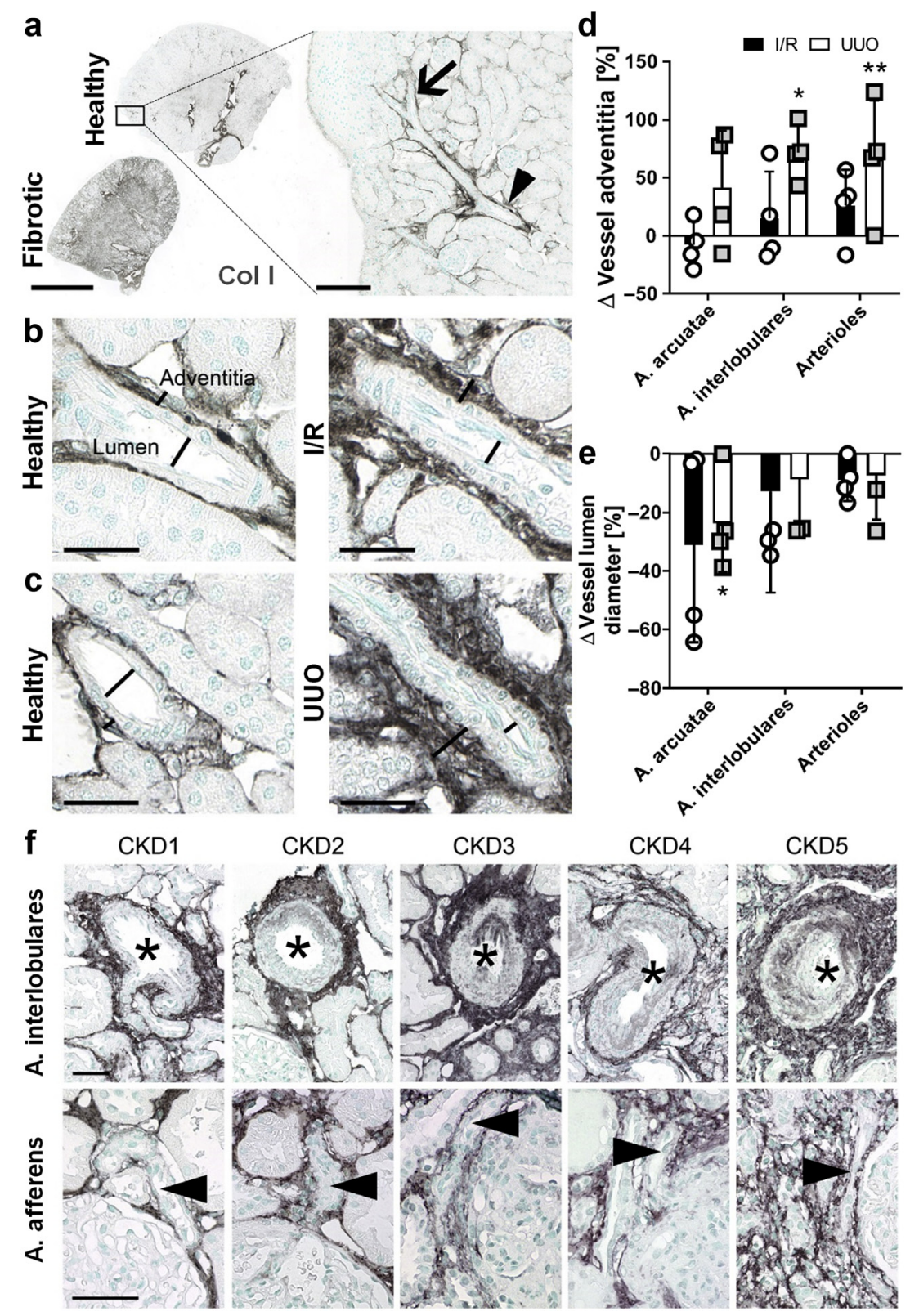

Figure 3 | Perivascular fibrosis is a constant feature of renal fibrosis in animal models and patients. (a) Localization and expression of collagen type I (Col I) in healthy and fibrotic renal tissues. The arrowhead points at the arteria arcuatae, and the arrow indicates the arteria (A.) interlobulares. Bar $=2 \mathrm{~mm}$. (b-e) Col I is upregulated during renal fibrosis in both ischemia-reperfusion injury (I/R) (b) and unilateral ureteral obstruction (UUO) (c) not only in the interstitium but also in the thickened adventitia of arterial vessels (d). Vessels showed a reduced vessel lumen diameter throughout the entire renal artery branches (e). Bar $=25 \mu \mathrm{m}$. (f) Enhanced Col III deposition in perivascular areas was also a common feature in human biopsies and increased with increasing chronic kidney disease (CKD) stage. Asterisks indicate the vessel lumen of arteria interlobulares, and arrowheads mark arteria afferens. Bar $=50 \mu \mathrm{m}$. Data are mean \pm SD of 4 mice per group. ${ }^{*} P<0.05,{ }^{* *} P<0.01$ fibrotic versus healthy kidneys. To optimize viewing of this image, please see the online version of this article at www.kidney-international.org.

\section{DISCLOSURE}

All the authors declared no competing interests.

\section{ACKNOWLEDGMENTS}

The authors gratefully acknowledge the technical assistance of Marie Cherelle Timm and Simon Otten in histopathology and of Michael Vogt in 2-photon microscopy. Image templates provided by Servier Medical Art were used for preparing the graphical abstract.
This study was supported by the German Research Foundation (DFG: SFB/TRR57, SFB/TRR219, SFB1066, BO3755/3-1, BO3755/6-1, GR5027/2-1, and RTG2375), the German Ministry of Education and Research (BMBF: STOP-FSGS- 01GM1901A), the European Research Council (ERC: StG-309495 and PoC-813086), the European Union (EU-EFRE: European Fund for Regional Development: I3-STM 0800387), the Interdisciplinary Centre for Clinical Research at RWTH (IZKF K7-3, E7-6, and 03-2), and the RWTH START program (09/15, $124 / 14$, and $152 / 12$ ). 


\section{SUPPLEMENTARY MATERIAL}

Supplementary File (PDF)

Figure S1. Optical imaging of collagen deposition in renal fibrosis using Cy7 fluorophore-labeled CNA35.

Figure S2. Characterization of collagen deposition in fibrotic kidneys. Figure S3. CNA35 co-localizes with collagen fibers in human kidney biopsies.

Figure S4. TPLSM-based characterization of CNA35 distribution in kidneys.

Supplementary Methods. Kidney fibrosis animal models; human kidney samples, histology, and microscopy analysis; renal histology, immunohistochemistry, and immunofluorescence; and 2-photon laser scanning microscopy and fluorescence microscopy.

\section{Supplementary References.}

\section{REFERENCES}

1. Djudjaj S, Boor P. Cellular and molecular mechanisms of kidney fibrosis. Mol Aspects Med. 2019;65:16-36.

2. Klinkhammer BM, Goldschmeding R, Floege J, et al. Treatment of renal fibrosis - turning challenges into opportunities. Adv Chronic Kidney Dis. 2017;24:117-129.

3. Baues M, Dasgupta A, Ehling J, et al. Fibrosis imaging: current concepts and future directions. Adv Drug Deliv Rev. 2017;121:9-26.

4. Sun $\mathrm{Q}$, Baues $\mathrm{M}$, Klinkhammer BM, et al. Elastin imaging enables noninvasive staging and treatment monitoring of kidney fibrosis. Sci Transl Med. 2019;11(486).
5. Krahn KN, Bouten CV, van Tuijl S, et al. Fluorescently labeled collagen binding proteins allow specific visualization of collagen in tissues and live cell culture. Anal Biochem. 2006;350:177-185.

6. Boerboom RA, Krahn KN, Megens RT, et al. High resolution imaging of collagen organisation and synthesis using a versatile collagen specific probe. J Struct Biol. 2007;159:392-399.

7. Chen J, Lee SK, Abd-Elgaliel WR, et al. Assessment of cardiovascular fibrosis using novel fluorescent probes. PLoS One. 2011;6:e19097.

8. Kramann R, Goettsch C, Wongboonsin J, et al. Adventitial MSC-like cells are progenitors of vascular smooth muscle cells and drive vascular calcification in chronic kidney disease. Cell Stem Cell. 2016;19: 628-642.

9. Kramann R, Schneider RK, DiRocco DP, et al. Perivascular Gli1 ${ }^{+}$ progenitors are key contributors to injury-induced organ fibrosis. Cell Stem Cell. 2015;16:51-66.

10. Desogere P, Tapias LF, Hariri LP, et al. Type I collagen-targeted PET probe for pulmonary fibrosis detection and staging in preclinical models. Sci Transl Med. 2017;9(384).

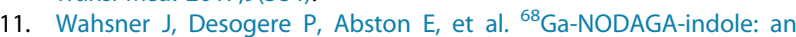
allysine-reactive positron emission tomography probe for molecular imaging of pulmonary fibrogenesis. J Am Chem Soc. 2019;141:55935596.

12. Kunjachan S, Gremse F, Theek B, et al. Noninvasive optical imaging of nanomedicine biodistribution. ACS Nano. 2013;7:252-262.

13. Gremse F, Doleschel D, Zafarnia $S$, et al. Hybrid $\mu C T-F M T$ imaging and image analysis. J Vis Exp. 2015;100:e52770.

14. Gremse F, Stark M, Ehling J, et al. Imalytics preclinical: interactive analysis of biomedical volume data. Theranostics. 2016;6:328-341. 